\title{
Foot-flat period estimation during daily living situations of asymptomatic and lower limb amputee subjects
}

\author{
B. Dauriac ${ }^{\mathrm{a}, \mathrm{b}}\left(\mathbb{D}\right.$, X. Bonnet $^{\mathrm{b}}$, C. Villa ${ }^{\mathrm{a}, \mathrm{c}}$, H. Pillet ${ }^{\mathrm{a}}$ and F. Lavaste $^{\mathrm{a}}$

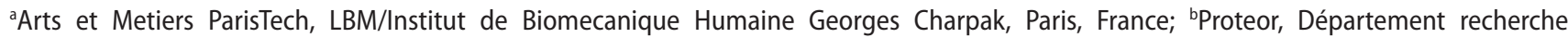 \\ et développement, Seurre, France; 'CERAH, Institution Nationale des Invalides, Woippy, France
}

KEYWORDS Gait; slope; temporal parameter; amputation; prosthetic foot

\section{Introduction}

Walking in various situations is a challenging task for people with a lower limb amputation. Walking upslope and downslope requires a larger ankle range of motion than waking on a level ground. Most of prosthetic feet do not include an ankle joint. The ankle mobility is obtained via the deformation of a composite structure or via rubbers. The range of motion of the "ankle-foot" component is directly linked to the stiffness of the structure and to the load applied on the prosthesis. In ramps, prosthetic "ankle-feet" present a lack of dorsiflexion when going up and a lack of plantar flexion when going down (Williams et al. 2009).

A decreased "ankle-foot" range of motion results in a reduced foot-flat period (FFP) which can induce instability. New systems were proposed to allow ankle-foot prostheses to adapt to slopes (Sup et al. 2009; Williams et al. 2009; Fradet et al. 2010).

Foot flat evaluation during different situations within the asymptomatic population could help to define a target for prosthetic design. This parameter could also be a very interesting tool for orthoprosthesist to give a feedback of the fitting of the prosthesis to the patient (Agrawal et al. 2009). A good adaptation of the prosthetic foot to the ground should result in a longer FFP and traduce the security of the subject on its prosthesis during stance phase. In the same way, a correct toe clearance during the swing phase will correspond to a contralateral side FFP close to normal. Actually, amputee people often demonstrate vaulting during swing phase showing their fear to stumble and fall. The evaluation of this parameter is all the more interesting for comparison purpose as it is not well taken into account during nowadays prosthesis design (Williams et al. 2009). Besides, FFP can be determined from on board measurements (Mariani et al. 2013) in real life conditions. However, for the moment, there are no reference data of FFP available in the literature.
The aim of the study is to quantify FFP in asymptomatic and amputee gait during level and slope walking.

\section{Methods}

Nineteen asymptomatic subjects (Control; 4F/15H mean \pm standard deviation, age: $37 \pm 17$, height: $176 \pm 9 \mathrm{~cm}$, mass: $71 \pm 15 \mathrm{~kg}$ ), eight transfemoral amputee men (TF; age: $41 \pm 10$, height: $178 \pm 6 \mathrm{~cm}$, mass: $81 \pm 3 \mathrm{~kg}$ ), and eight transtibial amputee men (TT; age: $52 \pm 10$, height: $177 \pm 5 \mathrm{~cm}$, mass: $88 \pm 13 \mathrm{~kg}$ ) all using a K-3 Medicare foot and a C-leg knee for the TF. Each subject was equipped with 54 markers to record segmental and articular kinematics of the body with a motion analysis optoelectronic system (Vicon V8i, UK) sampled at $100 \mathrm{~Hz}$. Markers were positioned on specific anatomical landmarks in accordance with the protocol described by Pillet et al. (2014). The subjects were asked to walk at self-selected speed on a level pathway, a 5\% slope and a $12 \%$ slope back-and-forth several times. According to Perry, mid-stance in able-bodied gait on level ground lasts from $12 \%$ to $31 \%$ of gait cycle (Perry 1992). It was assumed for all populations and situations that at the middle of this phase ( $20 \%$ of the gait cycle), the foot was stationary. FFP was computed as the time interval in which foot angle in the sagittal plane lied between $\pm 1.25^{\circ}$ around its orientation at $20 \%$ of gait cycle. This interval has been determined by an interative processes to have results in accordance with the literature for asymptomatic population in level walking. FFP was computed as a percentage of stance phase to normalize data among subjects. Statistical analysis was performed using an ANOVA test to determine significant differences on FFP between situations, groups, and sides (amputee and sound). When the ANOVA identified a difference between groups, post hoc Tukey's tests were utilized to demonstrate where the statistical differences had occurred. Normality and variance equality have been verified, respectively, using a Kolmogorov-Smirnov test and a Bartlett test beforehand. 


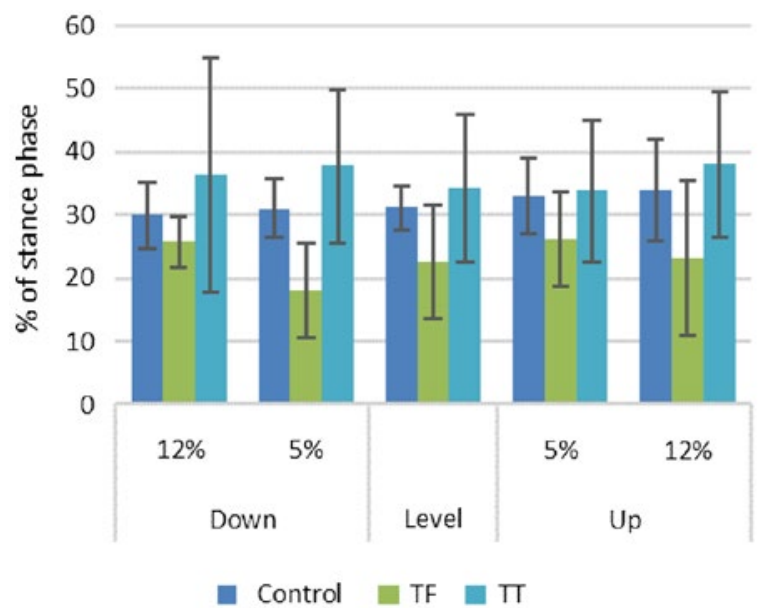

Figure 1. Mean contralateral side FFP during slope and level walking for TF and TT subjects compared to control.

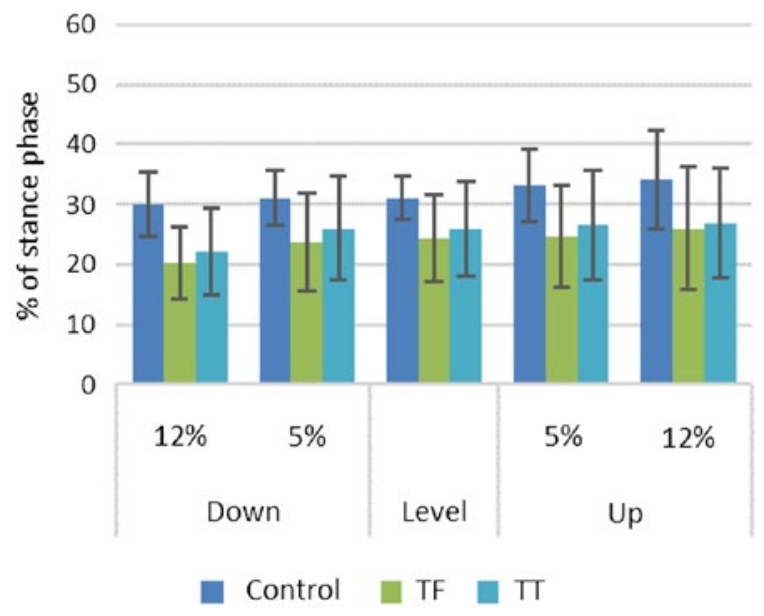

Figure 2. Mean prosthetic side FFP during slope and level walking for TF and TT compared to control.

\section{Results and discussion}

Results (Figures 1 and 2) are in accordance with Perry (30\% of the stance phase) for level walking gait in control group (Perry 1992) but lower than Mariani's results: $44 \%$ for the same population and situation (Mariani et al. 2013). Control group than prosthetic side FFP of both amputee groups. Contralateral FFP of TF was shorter than control FFP independently from the situation.

On the contrary, significant longer FFP have been found for the contralateral limb compared to the prosthetic one of TT group.

Amputee groups showed more variability than the asymptomatic one, which is in favor of a larger variety of strategies. On the contralateral side, FFP in TF were shorter in all situations than in TT and control groups probably due to vaulting gait to secure swing phase of prosthetic limb. On the prosthetic limb, both amputee groups had shorter FFP than controls. This might correspond to a lack of mobility of the prosthetic ankle already reported in the literature (Williams et al. 2009). The decrease of FFP of the pros- thetic foot during slope descent is in accordance with the non-adaptive stiffness of prosthetic energy storing feet already reported as a problem in slope descent (Pillet et al. 2014). Further studies should investigate more in detail the link between FFP and prosthetic ankle stiffness. A longer FFP should help lower limb amputee subjects to have a more symmetrical gait and a more stable stance phase.

This computation method could also be implemented for stair locomotion where an important decrease of the ankle stiffness has been identified for asymptomatic subjects (Pillet et al. 2014) to quantify the impact of the constant stiffness of the prosthetic foot on FFP. This could also be implemented for on-board measurements in daily living situations as it has already been proposed for level walking (Mariani et al. 2013).

\section{Conclusions}

FFP is a quick and easy-to-do method that could lead to insight in the adaptation of each patient to the situation and their prosthetic component. This study gives reference FFP for asymptomatic gait in slope as well as for TF and TT population using K-3 Medicare ankle-foot prosthesis. These results could be used as improvement assessment for future prosthesis design.

\section{Acknowledgements}

The authors wish to thank all the participants of this study.

\section{Funding}

This work was supported by the French National Research Agency [grant number ANR-292 2010-TECS-020]

\section{Orcid}

B. Dauriac (D) http//orcid.org/0000-0003-1804-862X

\section{References}

Agrawal V, Gailey R, O’Toole C, Gaunaurd I, Dowell T. 2009. Symmetry in external work (SEW): a novel method of quantifying gait differences between prosthetic feet. Prosthet Orthot Int. 33:148-156.

Fradet L, Alimusaj M, Braatz F, Wolf SI. 2010. Biomechanical analysis of ramp ambulation of transtibial amputees with an adaptive ankle foot system. Gait Posture. 32:191-198.

Mariani B, Rouhani H, Crevoisier X, Aminian K. 2013. Quantitative estimation of foot-flat and stance phase of gait using foot-worn inertial sensors. Gait Posture. 37:229-234.

Perry J. 1992. Gait analysis: normal and pathological function. Thorofare (NJ): SLACK.

Pillet H, Drevelle X, Bonnet X, Villa C, Martinet N, Sauret C, Bascou J, Loiret I, Djian F, Rapin N, et al. 2014. APSIC: training and fitting amputees during situations of daily living. IRBM. 35:60-65.

Sup F, Varol HA, Mitchell J, Withrow TJ, Goldfarb M. 2009. Self-contained powered knee and ankle prosthesis: initial evaluation on a transfemoral amputee. IEEE Int Conf Rehabil Robot. 2009:638-644.

Williams RJ, Hansen AH, Gard SA. 2009. Prosthetic ankle-foot mechanism capable of automatic adaptation to the walking surface. J Biomech Eng. 131:035002. 